\title{
Un profilo professionale di altri tempi: il fondachiere
}

\author{
Matilde Paoli
}

PUBBLICATO: 05 NOVEMBRE 2019

\section{Quesito:}

Vari lettori ci chiedono quale sia il significato del termine fondachiere; alcuni di loro vorrebbero conoscere cosa si intendesse con questo termine nel I930; più in particolare ci è stato chiesto cosa esso indicasse nel testo del Regio Decreto i8 giugno I931, n. 773.

\section{Un profilo professionale di altri tempi: il fondachiere}

F ondachiere è (con fondacaio, fondacaro o sporadicamente fondachista) uno dei derivati del termine fóndaco, prestito dall'arabo funduq 'alloggiamento per mercanti', a sua volta derivato del greco pandok(h)eion 'albergo' (DELI, L'Etimologico s.v.). La prima attestazione di fondaco in un volgare italiano risale (nella forma fontego) ai primi anni del XIII secolo (I207-I208) in un volgarizzamento di area veneta conosciuto come Patto del Soldano di Aleppo (cfr. TLIO s.v.). Fondaco e varianti secondo il TLIO risultano presenti nello stesso XIII secolo anche in testi toscani, in particolare senesi, pisani e fiorentini; nel secolo successivo si trovano attestazioni in documenti lucchesi e pistoiesi, e poi veneziani, romani, perugini e siciliani.

Troviamo la prima attestazione di fondachiere nel pisano Breve dei consoli della Corte dell'Ordine de' Mercatanti dell'anno MCCCXXI e altre ancora risalenti alla prima metà del secolo XIV in testi di area pisana e fiorentina. Un po' più antica la prima attestazione di fondacaio nel Breve dell'Arte della lana di Pisa datato i304. Infine troviamo per la prima volta la forma fondacaro in un testo siciliano anch'esso della prima metà del XIV secolo. Soltanto i primi due termini sono registrati nel Vocabolario degli Accademici della Crusca: fondacaio già nella II edizione (I623), mentre fondachiere appare nella III (I69I).

Queste tre voci, costruite applicando alla base fondaco esiti diversi del suffisso latino -arius (cfr. le schede Non sempre un portiere è un portinaio, Verduriere o verduraio? Verduraio o verdumaio?, Fiorista/fioraio e gelatiere/gelataio: professioni diverse o no?), si riferiscono a una persona la cui attività è legata al significato assunto da fondaco in luoghi e tempi diversi.

Dato il rapporto con il commercio e con la sua regolamentazione (evidente anche dal carattere delle prime opere che testimoniano le voci) possiamo trovare una rassegna dei valori assunti dal termine in un'opera specialistica, il Dizionario del linguaggio italiano storico ed amministrativo di Giulio Rezasco di cui diamo una sintesi [nostri i neretti e i commenti tra parentesi quadre]:

FONDACO e, secondo i dialetti, FONDICO, FONTICO, FONDEGO, sust.

I. Dogana [questo valore è riscontrato in testi di autori toscani a partire dal Decameron di Boccaccio e anche in testi a carattere tecnico senesi e pisani]

II. In Pisa, in Fermo, e forse in altri luoghi, ove il Fondaco della Dogana non era, o non bastava, Stanza terrena, talora con sopra altre stanze, condotta da privato, il quale per mercede tassata dagli Statuti la concedeva in Pisa a' mercanti cittadini of forestieri, che vi riponessero lor mercanzie e ve le vendessero, ma egli non poteva riporvi le sue; e coll'obbligo di tenere i registri di quelle mercanzie e delle vendite a disposizione de' Consoli de' Mercanti e di ubbidire alle loro prescrizioni; in Fermo, colla facoltà a' mercanti, come facevano nelle Dogane pubbliche, di vendervi le loro merci senza dazio alcuno, purché esse non si fermassero nella cittá, contado e distretto; qualche cosa di simigliante ai Punti franchi de' moderni [...]

III. Gabella del Fondaco o Fondaco semplicemente. Particolarmente in Sicilia ed in Puglia, gabella imposta 
dallimperatore Federigo nel 1220 [e poi gabella sulle merci in ingresso a Messina o a Napoli]

IV. Grande casamento, o Ceppo di case che per concessione del Signore del luogo, ed a fine di agevolare lo spazio delle derrate e merci di piu paesi, ove i mercanti di Nazione forestiera raunavano e tenevano sotto la fede pubblica lor mercanzie, scritture e danari, e vi esercitavano lor traffici; ed ove, almeno in qualche luogo e tempo abitavano altresi gli stessi mercanti e il loro Consolo. [È la base del ghetto: una sorta di area circoscritta ai mercanti di una determinata origine: cosi a Venezia il Fontego dei Tedeschi]

V. VI. [sono il Visdomino del fontego dei Tedeschi a Venezia e il nome di una via di Siena]

VII. Emporio

VIII. Pubblico granaio

IX. Specialmente nel Veneziano, e nell'Istriano, il Magazzino o la Canova, ove si riponevano le biade e le farine del Comune provenienti da terratici, e simili rendite pubbliche; le quali biade e farine il Fonticaro o Fondacajo vendeva di mano in mano a' cittadini...

X. Vero Monte frumentario in Trieste, ove provvide all'Annona prima co' danari del Comune e poscia co' suoi; in Verona, ove aveva entrate proprie, fornite dalle Arti e da elemosine, ed ove esso si apriva nelle carestie, somigliante al Monte della Farina di Modena; ed in Treviso, ove era governato da Conservatori eletti dalle Arti e da elemosine.

XI Nel Napoletano, anche Camera del sale

XII In Modena, la Stanza ove gli Ufficiali de' Monti tenevano la residenza. [s.v. si dice che il Fontico poteva essere anche "del Monte di Pietà"]

$[\ldots]$

Da questa rassegna delle realtà diverse indicate con il termine fondaco possiamo dedurre che la figura delfondachiere (o fondacaio, fondacaro, fonticaro, ecc.) poteva assumere diversi profili e svolgere mansioni diverse: poteva essere l'esattore di un canone dai mercanti le cui merci ospitava e custodiva nel locale assegnatogli - e in questo caso doveva documentare il traffico di merci e renderne conto; poteva essere il distributore di biade e farine ai concittadini in tempi di carestia o il supervisore di un ufficio doganale; poteva avere l'incarico di gestore del locale in cui il sale veniva depositato, registrato, messo in vendita; o poteva essere il gestore di un'agenzia di prestiti su garanzia di oggetti dati in pegno.

Ciò che accomuna questi profili è il rapporto con l'amministrazione pubblica alla quale il gestore del fondaco era tenuto a rendere conto, diventando come un intermediario tra essa e i mercanti o i cittadini.

Ai valori storici della voce fondaco si deve poi aggiungere un ulteriore significato ottocentesco attestato dal GDLI come regionalismo, che potremmo definire di area campana, visti i riferimenti a Napoli di tutti i testi citati [nostri il neretto e lo scioglimento dei rimandi bibliografici]:

Seminterrato, adibito ad abitazione poverissima. Periodici popolari del Risorgimento, I818-1870, I-60: Gittate gli occhi, di grazia, per entro que' miseri abituri, chiamati a Napoli fondi e altrove fondachi, ché le son caverne e non altro. Matilde Serao, Il ventre di Napoli, I884, in Opere, a cura di P. Pancrazi, I944, vol. I, p. I098: Le quattro viottole cieche che salgono da Santa Lucia verso la collina, valgono i 'fondaci' del quartiere Mercato, per il luridume. B. Croce, Storia d'Italia dal 1871 al 1915, 1928, p. 85 dell'ed. 1959: Le misere condizioni igieniche e morali del popolino di Napoli, i «fondachi», i «bassi», la «camorra», furono rese note dal Fucini. Massimo Bontempelli, Stato di grazia, 1934, p. I35 dell'ed. 1942: Napoli li diverte [i forestieri] con i bassi dietro Porta Capuana, i brulicami di avanzi umani vivi rovesciati a ceste giù per i fondachi.

Questo valore sembra collegabile a quanto testimoniato nella lessicografia dialettale setteottocentesca di area napoletana: nel vocabolario di Galiani (I789) funneco [sic] è "fondaco, e casa matta, lat. fornix propria della plebe, e delle bagasce", mentre in quello di D'Ambra (I873) fünneco è "Fondaco [...] 2. Vico rotto, vietta cieca, Ronco [...] 3. Chiassuolo, o Corte, dove abitano artieri, ed operai diversi" e funnachiero "Abitante di fondachi, che son maniera di Ronchi cui si entra per un arco 2. 
detto di donna è parola d'oltraggio, Ciana, Donnaccia. 3. Rivenditore a ritagli. [ronco "Strada che non ha uscita"; a ritaglio "Al minuto" in Tommaseo-Bellini]

Riportiamo altre testimonianze dal panorama della lessicografia dialettale. Al Nord della penisola (come notato da Novelli 1989 alla voce fondichiere 'proprietario, gestore di un fondaco, di una bottega') il piemontese fondiché registrato nei dizionari di area e il milanese fondeghée presente nel vocabolario di Cherubini (I8I4) hanno il solo significato di 'droghiere'. Il bergamasco fondeghér nel vocabolario di Tiraboschi (I862) viene genericamente equiparato a fondachiere 'chi tiene il fondaco' e fondacajo 'padrone del fondaco', mentre per il genovese il dizionario di Casaccia (I85I) testimonia fondeghé "Quegli che tien bottega o magazzino e rivende il vino a minuto" e fondego "Luogo dove si vende il vino a minuto". Infine Ferrari (I820) per il bolognese fundghir segnala il valore particolare di "Mercante di legnami. Colui che incetta legnami, mattoni, cannucce, calcina, e simili materiali per uso di fabbricare e li vende al minuto".

Per il Sud, oltre a quanto già visto per l'area napoletana, disponiamo delle testimonianze novecentesche di Giammarco (I968-I990) che per abruzzese e molisano riporta fónacha [e varianti] "fondaco, luogo di deposito, f. de le rana granaio // bottega dove si vende la mercanzia", fúnacha "negozio dove si acquista un po' di tutto" e funachjére "venditore di tessuti". Per il Salento Rohlfs (1956-196I) testimonia solo la forma funachira che equipara a fondacaio. Lo stesso autore dà informazioni più dettagliate nel suo Dizionario dialettale delle tre Calabrie (1932) dove fúndacu le varianti] indica "fondaco, bottega, magazzino nel pianterreno di una casa, rivendita di sale e tabacco", fundacaru è il "padrone del fondaco" e fundacheri è "padrone del fondaco o rivenditore di sale e tabacco". Per la Basilicata Bigalke (I980) dà fünnəkə per "fondaco e anche bottega". Più articolato il valore testimoniato per il siciliano da Giorgio Piccitto (vol. II 1985): fünnacu vale "Stallaggio, stalla pubblica con possibilità di pernottamento anche per le persone. 2. locanda, osteria, 3. mercato"; funnacaru e fundacaru "proprietario o gestore di un fondaco 2. oste 3. persona volgare"; fundacara "donna sguaiata, di facili costumi".

Questa molteplicità, testimoniata per l'antico e almeno in parte sopravvissuta nei dialetti, non appare appieno nella lessicografia di lingua: nel Tommaseo-Bellini (I86I-I879) fondachiere (cosi come il fondacaio dato ormai come desueto) è semplicemente un "Maestro di fondaco" e il fondaco indica una realtà relativamente complessa:

I. Luogo dove sono deposte merci di molti o d'un solo, per venderle quivi, o altrove portarle a usi commerciali.

2. Bottega dove si vendono panni e drappi.

3. [M.F.] Chi ha fondaco.

4. Per Parte della città, dove abitano tutti uniti e separati dagli altri quelli di alcuna nazione forastiera.

5. Trasl. Gran copia, Grande abbondanza di checchessia.

Nella pressoché coeva V edizione del Vocabolario degli Accademici della Crusca il primo significato di fondaco è "Bottega, dove si vendono a ritaglio, e anticamente anche in di grosso, panni di lana e drappi." Poi si aggiunge "In senso generico, per Bottega, Magazzino, Luogo o Stanza ove si tengono le mercanzie e le grasce Igrascia "qualsivoglia cosa che serva al vitto degli uomini", spec. cereali, vino e olio] da vendersi, o da farne commercio". Si riportano infine gli antichi significati di "Grande edifizio, concesso in un porto di mare ai mercanti di alcuno stato o nazione per uso di deporvi e di vendervi in di grosso le lor mercanzie, e di alloggiarvi essi stessi, pagando un certo diritto" e "Dogana in alcun porto di mare, o Edifizio con magazzini, per uso di deporvi e vendervi, pagando certi diritti, le mercanzie portate di fuori". 
Almeno nel panorama culturale di riferimento della V Crusca il fondaco è ormai un negozio in cui si vendono soprattutto tessuti, oltre che un magazzino/deposito di merci varie da vendersi in di grosso ovvero "In grossa quantità, In grossa partita" modalità contrapposta alla vendita al minuto, corrispondente quindi a quel che oggi indichiamo con vendere all'ingrosso.

La semplificazione riscontrata nel XIX secolo si nota ancora di più nel XX secolo, periodo che interessa a chi chiede il valore assunto dal termine nel i930. Soffermiamoci quindi su quanto attestato nella lessicografia coeva.

Nella IV (I929-1930) e nella V (1935-1936) edizione del Vocabolario della lingua italiana di Nicola Zingarelli troviamo fondachiere, segnato con la crux a indicarne la desuetudine, per "Mercante, negoziante che tiene fondaco" e fondacaio, anch'esso desueto, che ha, al solito, valore leggermente diverso "Proprietario del fondaco, Mercante". L'unica cosa certa quindi è che si tratta di operatore commerciale. Per avere l'informazione completa occorre ancora una volta sapere cosa sia il fondaco: nelle stesse edizioni e anche nella prima (I9I7) Zingarelli lo definisce "Bottega dove si vendono tessuti al minuto", "Magazzino, Luogo di deposito di mercanzie" e, come obsoleto, "Albergo di mercanti in paesi stranieri, spec. sulle coste del Mediterraneo".

Difficile quindi rispondere con precisione in base alla lessicografia sia a chi chiede genericamente cosa si intendesse con fondachiere negli anni Trenta del Novecento, sia a chi vuol sapere cosa indicasse nel citato Regio Decreto I8 giugno I93I, n. 773, di cui, per chiarezza riportiamo il testo dell'articolo I28 con integrazione tra [] tratta dall'articolo i27:

Art. I28. I fabbricanti, i commercianti, gli esercenti e le altre persone indicate negli artt. I26 e I27 [Art. I27 comma r: I fabbricanti, i commercianti, i mediatori di oggetti preziosi, hanno l'obbligo di munirsi di licenza del Questore] non possono compiere operazioni se non con le persone provviste della carta di identità o di altro documento munito di fotografia, proveniente dall'amministrazione dello Stato.

Essi devono tenere un registro delle operazioni che compiono giornalmente, in cui sono annotate le generalità di coloro con i quali le operazioni stesse sono compiute e le altre indicazioni prescritte dal regolamento.

Tale registro deve essere esibito agli ufficiali ed agenti di pubblica sicurezza, ad ogni loro richiesta.

Le persone che compiono operazioni con gli esercenti sopraindicati, sono tenute a dimostrare la propria identità nei modi prescritti.

Lesercente, che ha comprato cose preziose, non può alterarle o alienarle se non dieci giorni dopo l'acquisto, tranne che si tratti di oggetti comprati presso i fondachieri o i fabbricanti ovvero all'asta pubblica.

Il testo, praticamente identico a quello dell'art. I29 del Regio Decreto del 6 novembre I926, n. I848, ci pare trovi un precedente più lontano nell'articolo 64I del Codice Penale di S.M. Il Re del Regno di Sardegna esteso alla Sicilia... del I86I:

64I. I gioiellieri, orefici, oriuolai, e qualsivoglia persona che attenda alla compra e vendita di gioie, ori, od argenti, gli ottonai, stagnaiuoli, calderai, rigattieri o ferravecchi, dovranno fare al Sindaco del Comune, o ad altra Autorità a ciò destinata, o in mancanza di questi al Segretario del Comune e in di lui assenza al Segretario del Giudice, una distinta e circostanziata dichiarazione di tutte le cose che compreranno o riceveranno in pegno, pagamento o permuta, oppure per vendere, esprimendone la quantità, qualità, ed altri connotati, ed il prezzo per cui avranno quelle avute, ed indicando altresì il nome, cognome, patria e condizione delle persone che gliele avranno vendute o rimesse. Tale dichiarazione debbe essere fatta entro ventiquattro ore dopo che avrà avuto luogo la vendita o rimessione. In caso di trasgressione, le persone suddette sono punite con multa estensibile a lire cento; e, se fossero recidive, col carcere per tre mesi, e colla sospensione dall'esercizio della loro professione.

Sono però eccettuate dall'obbligo della dichiarazione le robe che saranno comprate nei fondachi o 
negozi aperti (Libro II, Titolo IX Dei reati contro le persone e le proprietá, CAPO II Dei reati. contro le proprietà, Sezione III).

Analoghi testi, riferibili al I8I5 e al I770, si trovano in due precedenti raccolte di normative del governo dei Savoia.

Costanti in tutti questi documenti sono il riferimento a una merce particolare (gioie, ori, argenti che divengono cose o oggetti preziosi nel testo più recente); il riferimento alle transazioni che coinvolgono questa merce, lobbligo di tenere un registro con indicazioni precise sugli oggetti e sulla loro provenienza e l'esonero da quest'obbligo se la negoziazione è avvenuta "in fondachi o negozi aperti" o "presso i fondachieri o i fabbricanti ovvero all'asta pubblica" come nel testo del I93I.

In sostanza si parla di commercio di oro e gioielli di cui deve essere rintracciata la provenienza in modo da evitare il rischio di ricettazione. Il fondachiere (o il gestore del fondaco) è persona che può comprare, vendere o ricevere in pegno o in pagamento o permuta gioielli (probabilmente tra le altre merci che può trattare).

Il fondachiere citato nel regio decreto è da intendersi come gestore di fondaco (si ricordi che negli antecedenti sette e ottocenteschi del testo si parla appunto di merci acquistate nei fondachi). In questa prospettiva è utile ricordare quanto Rezasco riportava al punto XII per Modena a proposito del rapporto tra il fontico e il Monte di Pietà. Troviamo una testimonianza analoga nell'ottocentesco Regolamento per il Monte pio della cittá di Pistoia approvato da S. A.I. e R. con veneratissimo dispaccio dei 20 settembre 1832 (Pistoia, Stamperia dei Bracali, [1832]) in cui è evidente lo stretto rapporto tra i fondachisti a cui sono affidati gli "oggetti, che dai Particolari vengono impegnati, che custodisconsi nei Fondachi" e il Monte. In pratica i gestori del fondaco sono i garanti della custodia dei beni impegnati per ottenere prestiti.

In conclusione, riguardo a cosa si intendesse con fondachiere intorno al 1930 possiamo rispondere che in lingua comune il termine ha significato piuttosto generico e riferito a una realtà già all'epoca non più attuale, mentre nei dialetti che ancora usavano il termine (o altri derivati da fondaco) esso indicava realtà diversificate. La lingua del diritto ci offre una prospettiva storica molto articolata, ma che all'epoca non pare più molto attuale. Riguardo infine al Regio Decreto del i93i possiamo dire che il testo verosimilmente si riferisce all'uso del fondaco come deposito di oggetti preziosi acquistati o ricevuti in pegno o permuta di cui il gestore (il fondachiere) si rendeva garante. Rimane comunque difficile riferirlo alla realtà attuale delle normative vigenti che regolano la compravendita dell'oro e dei preziosi.

Nota bibliografica:

- Rainer Bigalke, Dizionario dialettale della Basilicata con un breve saggio della fonetica, un'introduzione sulla storia dei dialetti lucani e note etimologiche, Eidelberg, Winter Universitätsverlag, I980.

- Giovanni Casaccia, Vocabolario genovese-italiano, Genova, Pagano, I85I.

- Francesco Cherubini, Vocabolario milanese-italiano, Milano, Stamperia Reale, I8I4.

- Raffaele D'Ambra, Vocabolario napolitano-toscano domestico di arti e mestieri, Napoli, a spese dell'autore, I873.

- Claudio Ferrari, Vocabolario bolognese co' sinonimi italiani e franzesi, Bologna, Nobili, I820.

- Ferdinando Galiani, Vocabolario delle parole del dialetto napoletano che piu si scostano dal dialetto 
toscano con alcune ricerche etimologiche sulle medesime degli Accademici filopatridi, Napoli, Giuseppe Maria Porcelli, I789.

- Ernesto Giammarco, Dizionario abruzzese e molisano, Roma, Edizioni dell'Ateneo, I968-I99o.

- Silverio Novelli, Piemontesismi e francesismi in un dizionario del notariato ottocentesco, "Studi di Lessicografia italiana", I0, I989, pp. г25-270.

- Giulio Rezasco, Dizionario del linguaggio italiano storico ed amministrativo, Firenze, Successori Le Monnier, I88I.

- Gerhard Rohlfs, Vocabolario dei dialetti salentini: terra d'Otranto, München, Beck, I956-196I

- Gerhard Rohlfs, Dizionario dialettale delle tre Calabrie: con note etimologiche e un'introduzione sulla storia dei dialetti calabresi, Halle-Milano, Niemeyer-Hoepli, 1932.

- Antonio Tiraboschi, Vocabolario dei dialetti bergamaschi antichi e moderni, Bergamo, Fratelli Bolis, I862.

- Vocabolario siciliano, a cura di Giorgio Piccitto; [poi] a cura di Giovanni Tropea, Centro di studi filologici e linguistici siciliani, Opera del vocabolario siciliano (vol. II i985).

\section{Cita come:}

Matilde Paoli, Un profilo professionale di altri tempi: il fondachiere , "Italiano digitale", 2019, XI, 2019/4 (ottobre-dicembre)

DOI: $10.35948 / 2532-9006 / 2020.3257$

Copyright 2019 Accademia della Crusca

Pubblicato con licenza creative commons CC BY-NC-ND 